Mappemonde

Revue trimestrielle sur l'image géographique et les

formes du territoire

127 | 2019

Varia

\title{
L’Atlas des Régions Naturelles. Éric Tabuchi, Nelly Monnier
}

Laurent Jégou

OpenEdition

Journals

Édition électronique

URL : http://journals.openedition.org/mappemonde/1816

DOI : 10.4000/mappemonde.1816

ISSN : $1769-7298$

Éditeur

UMR ESPACE

Référence électronique

Laurent Jégou, «L'Atlas des Régions Naturelles. Éric Tabuchi, Nelly Monnier », Mappemonde [En ligne], 127 | 2019, mis en ligne le 01 juillet 2019, consulté le 15 septembre 2020. URL : http:// journals.openedition.org/mappemonde/1816

Ce document a été généré automatiquement le 15 septembre 2020.

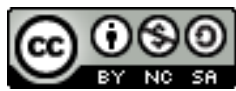

La revue Mappemonde est mise à disposition selon les termes de la Licence Creative Commons Attribution - Pas d'Utilisation Commerciale - Partage dans les Mêmes Conditions 4.0 International. 


\title{
L'Atlas des Régions Naturelles. Éric Tabuchi, Nelly Monnier
}

\author{
Laurent Jégou
}

L'Atlas des Régions Naturelles (ARN : https://atlasrn.fr/) est un titre qui fait s'arrêter le géographe dans ses déambulations sur le web, attiré par l'idée d'une présentation visuelle de régions. La lecture de la page d'accueil (figure 1) continue d'intriguer par la diversité des images qu'elle propose et son aspect épuré.

Figure 1. La page d'accueil du site

Ce site explique ce qu'est l'Atlas des Régions Naturelles, et qui en sont les auteurs. Découvrez une large sélection d'images et si vous voulez soutenir le projet, faites l'acquisition de photographies d'Eric Tabuchi, de peintures et d'écussons de Nelly Monnier. Vous pouvez aussi suivre nos actualités, lire notre journal de route ou souscrire à la newsletter.
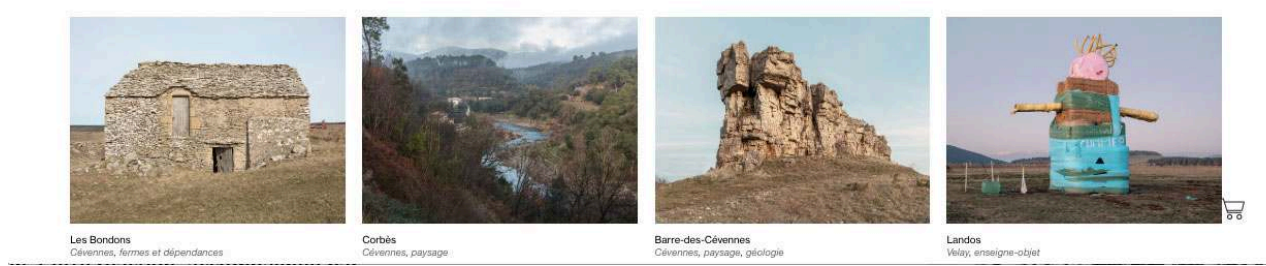

Source : ARN

2 Car il s'agit, avant tout, d'un projet artistique. Le principe déclaré est simple : réaliser 50 prises de vues dans les (presque) 500 "régions naturelles de France» (https:// fr.wikipedia.org/wiki/Région_naturelle_de_France), soit près de 25000 photographies, 
concernant les objets construits par l'homme (le bâti) et les paysages qui en sont le contexte. Cet atlas photographique se donne pour objectif de constituer une archive visuelle de ces régions naturelles, à terme organisé selon des critères géographiques et thématiques (type d'objet bâti, fonction). Le projet a commencé à produire des résultats au travers d'un site Internet, la publication d'ouvrages, l'organisation d'expositions. Le site, ouvert en 2017, est alimenté régulièrement par de nouvelles photographies, il en compte près de 180 au moment d'écrire ces lignes, sur plusieurs régions disparates.

3 Le géographe intrigué poursuit sa découverte. Point de départ du projet, les « régions naturelles " (figure 2) n'ont rien de véritablement naturel, culturel ou officiel, car plusieurs définitions coexistent, selon les critères utilisés. Ici elles ont été choisies par les deux auteurs parce qu'elles découperaient le territoire en entités à taille humaine, dont l'exploration photographique de l'architecture et des paysages serait à la portée de la petite équipe du projet. Au premier abord, on pourrait les rapprocher des "petites régions agricoles » ou des "pays» historiques, c'est leur cohérence du point de vue architectural et du milieu naturel qui les justifierait. Les artistes ont donc commencé par produire la carte de ces régions (http://www.poursuite-editions.org/index.php?/ parutionspubli/carte-arn/), malgré les difficultés à les délimiter précisément :

"Cependant, même s'il est impossible d'en tracer les frontières exactes, leurs limites géologiques, historiques et culturelles persistent à dessiner, dans une sorte de tradition orale, les contours d'une géographie dont la vivacité demeure bien réelle. Après expérimentations, la trame que tissent ces pays, du fait de leurs dimensions réduites, de leurs caractéristiques géologiques propres, mais aussi de la relation affective qu'il est possible d'entretenir avec chacune d'elles, s'est révélée être la plus appropriée à la réalisation d'un tel projet. Cette carte qui les délimite sur la totalité du territoire français constitue donc le document de référence de l'ARN. »

Figure 2. Le travail pour produire la carte des régions naturelles de France

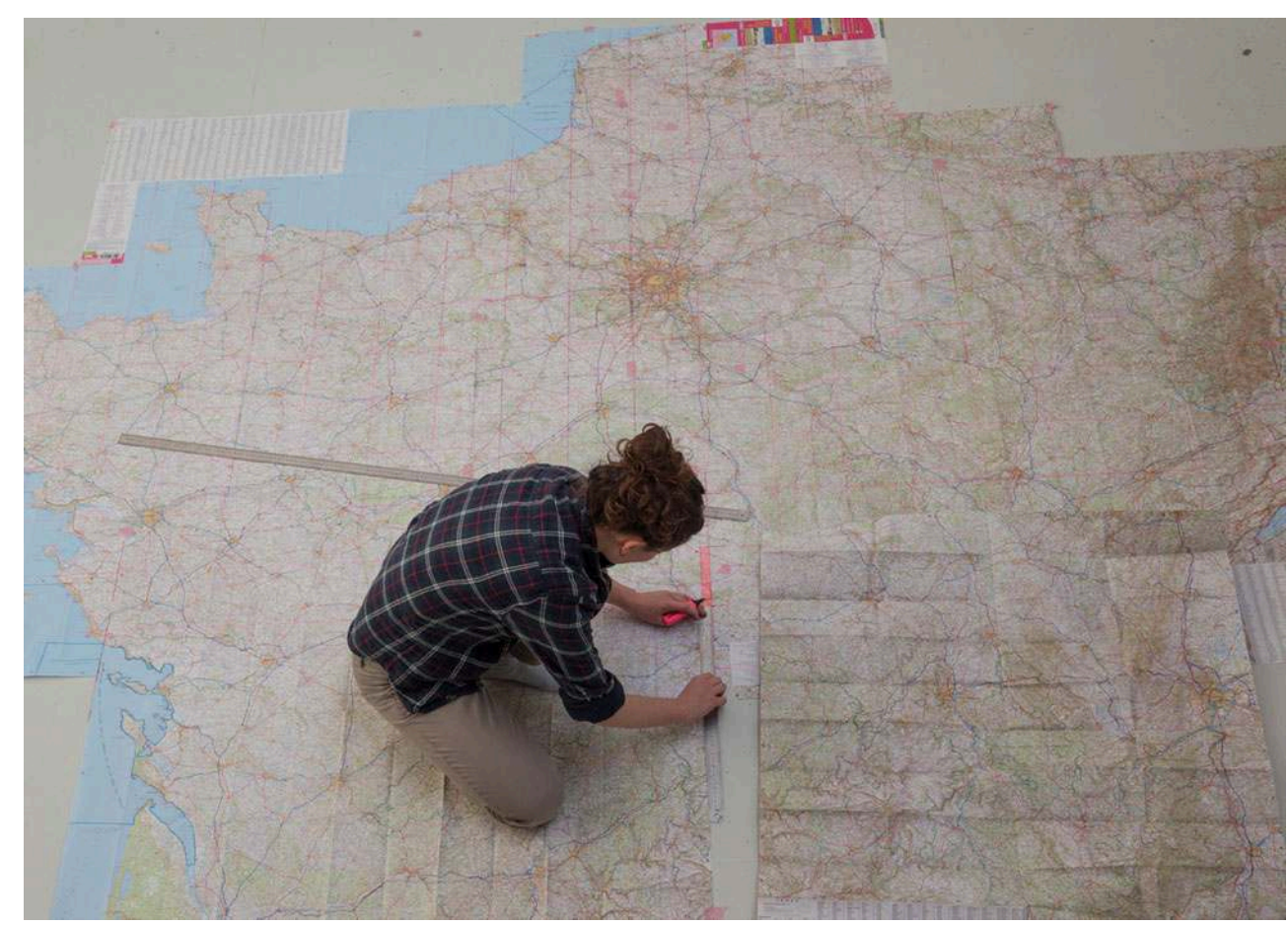

Source : ARN 
Cependant, en étudiant certains de ces découpages de plus près, on remarque tout d'abord le relatif éloignement entre les délimitations choisies et celles des régions agricoles ou historico-culturelles classiques en géographie. Ensuite, la carte présente des éléments surprenants, des choix de contenus assez étranges, aux critères difficiles à cerner (figure 3). Dans cet extrait de la carte que publient les éditions Poursuite, on remarque vite que l'ouest du Bassin Parisien comporte des éléments étonnants, des cours d'eau de petit gabarit sont dessinés (Iton, Epté), certaines petites villes semblent élevées au rang de capitales locales (Le Neubourg, Bourg-Achard), alors qu'il manque évidemment des références incontournables comme L'Eure ou Elbeuf et que des régions naturelles comme le Perche ne sont pas mentionnées. À y regarder plus sérieusement, on se rend compte que l'Iton figuré sur la carte est en fait l'Eure, la véritable Iton étant plus à l'ouest (elle coule à Évreux). Erreurs ou décalages, (licences) artistiques ? Volonté de jouer sur les codes graphiques de la carte classique pour cartographier des régions qui relèvent plus du domaine du vécu, du sensible, voire de l'imaginaire? La photographie de l'artiste dans la carte (figure 2), avec ses instruments de mesure, évoque naturellement l'imaginaire du/de la cartographe, artiste et artisan. Cependant, sur cette photographie, celle-ci semble produire un quadrillage régulier, assez éloigné de l'idée de régions "naturelles ", et la Corse est oubliée... (sans parler de l'Outre-Mer, source de paysages de valeur s'il en est).

Figure 3. Extrait de la carte des régions naturelles

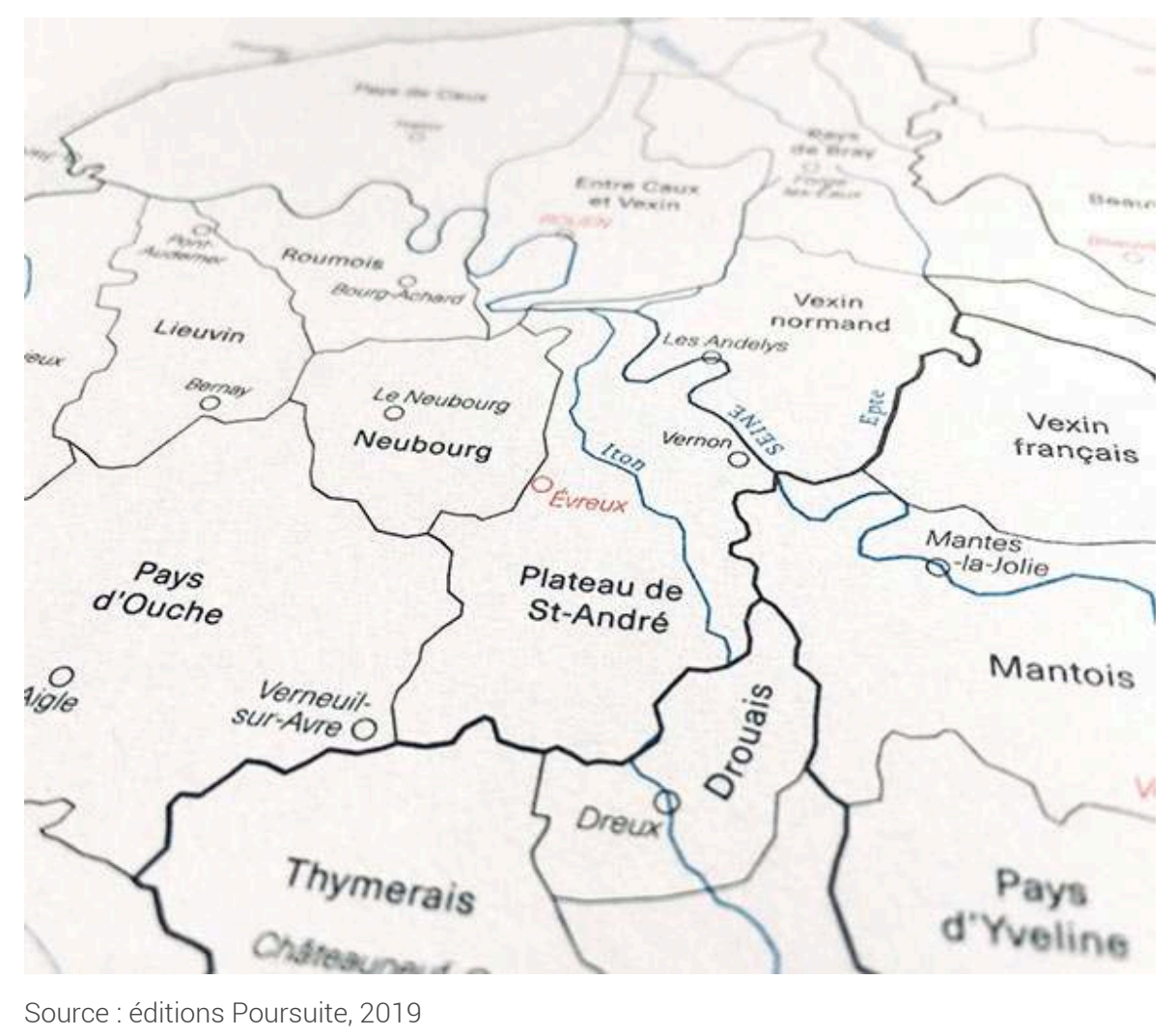

Dans la même veine, le choix des sujets de la photographie semble lui aussi parfois assez éloigné d'une vocation encyclopédique ou archivistique, qui supposerait une grille systématique: les bâtiments, monuments, enseignes, empilements choisis sont loin d'être les plus évocateurs ou représentatifs de leur région. On repère, sans vouloir 
être ici exhaustif, des piles de bottes de foin, des tas de bois, du linge qui sèche... On finit par se demander si l'un des objectifs des artistes n'est pas de provoquer ce décalage de signification entre ce que le titre annonce, l'exercice classique de l'atlas géographique et les matériaux qu'ils nous proposent, photographies et carte associées. Cet écart sémiotique constitue une des bases de la rhétorique visuelle, au sens du Groupe $\mu$ (1992), qui fait naitre le sentiment, l'émotion esthétique, lors de sa reconnaissance. Parce que l'on se rend compte, surpris, que l'œuvre ne nous présente pas ce qui était attendu, malgré tous les éléments qui pouvaient nous le faire croire $a$ priori, on en retire une émotion artistique, celle de la découverte, qui stimule l'imagination et encourage la lecture. L'autre attrait de cette œuvre, c'est sa dimension picturale et documentaire, démultipliée, dans un souci du cadrage et des lumières qui permet la comparaison de suites d'images, sur les formes, les structures et les fonctions des espaces représentés.

7 Le même décalage apparaît dans les écussons des « régions naturelles " produits en broderie par Nelly Monnier (sur son site propre : https://nellymonnier.com/SHOP) : les régions en partie "inventées " par les artistes se voient illustrées par des emblèmes tout aussi neufs, tirés d'une interprétation de certaines caractéristiques locales. Ainsi, le Bugey, une région historique de l'Ain, est représenté par une vision violette de l'écorce des sapins, à la place du lion belliqueux du blason officiel (figure 4).

Figure 4. Le blason du Bugey selon l'ARN et selon I'héraldique
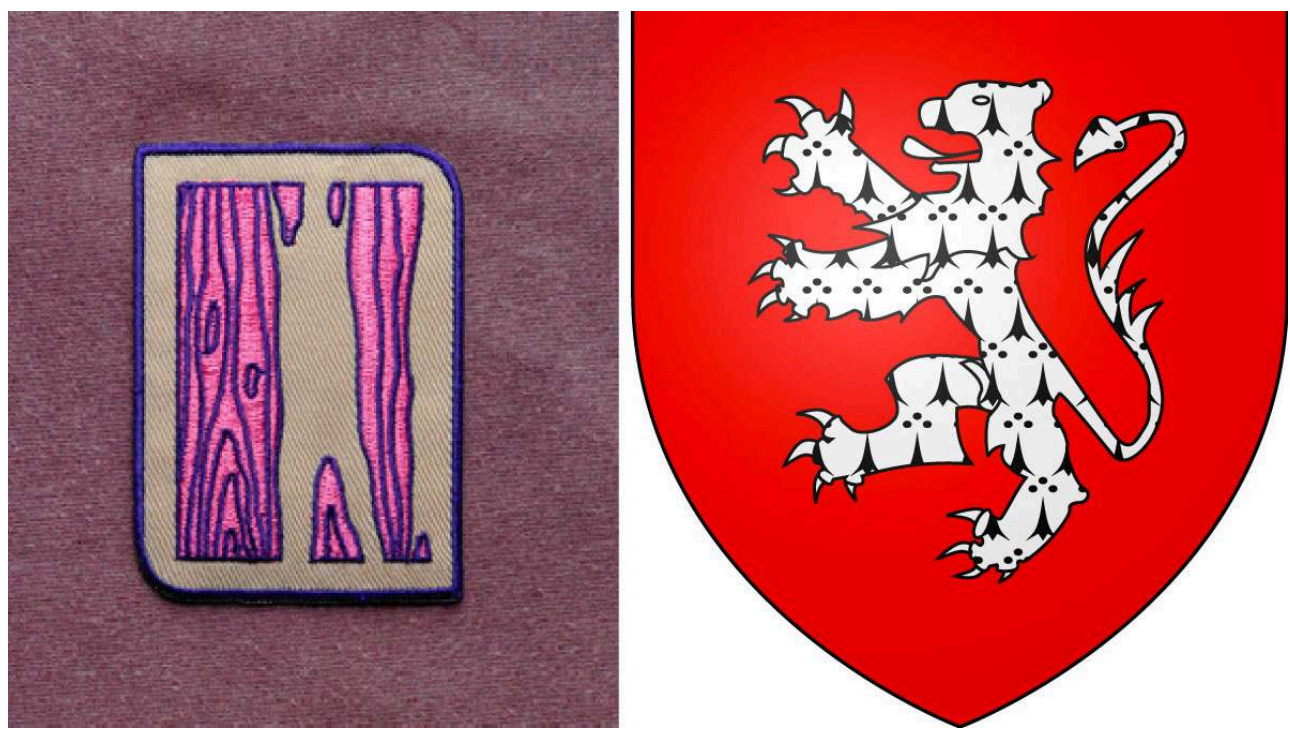

Sources : N. Monnier; Wikipedia (https://commons.wikimedia.org/wiki/File:Blason_pays_fr_Bugey.svg)

Les auteurs de l'ARN semblent ainsi présenter un tropisme envers les bâtiments vestiges, traces d'usages passés, en ruine ou abandonnés, ce qui enveloppe le projet d'une certaine aura de nostalgie. L'architecture traditionnelle est bien présente, mais relativement peu et au côté de types emblématiques du projet comme les châteaux d'eau, les ruines industrielles, les commerces anciens, les enseignes monumentales, les silos... Éric Tabuchi nourrit une certaine attirance, par ailleurs, pour les bâtiments du réseau électrique (auxquels il consacre un ouvrage spécifique en 2019, qui montre notamment les adaptations architecturales locales de ces constructions, visant à mieux les intégrer dans le paysage). Du point de vue de la photographie, le style choisi exprime la nostalgie et un certain décalage de la réalité : les couleurs sont assez 
neutres, les cadrages centrés, les lumières plutôt automnales, les lieux quasiment sans vie (figure 5).

Figure 5. Extrait du catalogue de l'ARN


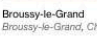

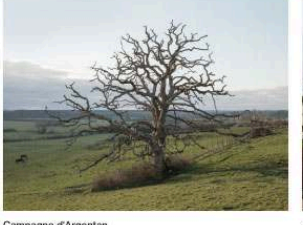

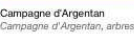

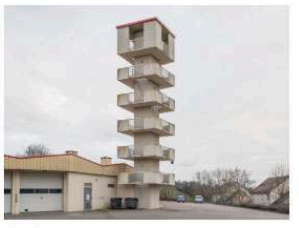

Charolus


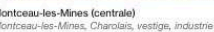
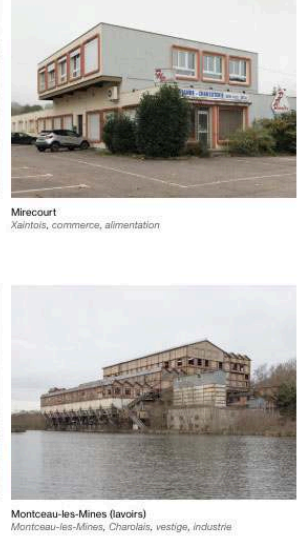

Source : ARN

Ne nous y trompons pas : l'ARN est un projet artistique, qui, s'il entretient des liens avec l'imaginaire géographique via l'ancrage de départ sur le concept de région naturelle, la photographie de paysages et la cartographie, va voir les auteurs sélectionner les sujets, bâtiments et paysages selon leur sentiment et leur jugement esthétique plutôt qu'en suivant une systématique ou une règle d'équilibre entre les thèmes ou les types. La présentation des vues sur le site, mélangeant les régions, les lieux, les types et les sujets, ne facilite pas la reconnaissance d'une "naturalité " régionale, d'une identité culturelle, c'est plutôt, à l'inverse, une certaine image de déréliction générale de l'œuvre humaine dans les paysages, du caractère presque absurde de l'inventivité et de l'originalité de ces constructions-vestiges, dans un monde déserté, qui ressort. Plutôt que la recherche de manifestations de l'activité humaine sur les territoires, c'est la mise en avant de traces d'une activité révolue, passives, qui a été choisie.

De manière plus scientifique et archivistique, ce projet rappelle d'autres travaux de photographie de territoires, «sérieux» cette fois-ci, notamment la Mission photographique de la Datar: https://missionphotodatar.cget.gouv.fr/mission (1984-1989), qui a vu une équipe d'une trentaine de photographes parcourir les territoires et amasser une collection de photographies de paysages pour «saisir un moment singulier de son évolution ", avant transformation irrémédiable (Le Toquin et Voisin, 2018). Il s'agissait alors d'une commande publique de photographie d'art, l'Atlas des Régions Naturelles est, lui, soutenu par le Centre National des Arts plastiques et le CAUE d'Indre-et-Loire.

11 Un parallèle peut aussi être tenté avec le travail plus récent de témoignage/ préservation des paysages $d u$ réalisateur et photographe Raymond Depardon, notamment «La France de Raymond Depardon » avec une grande exposition à la BNF en 2010. Ce projet était motivé par la même volonté de " guetter les traces de l'homme sur le territoire ", à l'aide d'un équipement particulier et selon un itinéraire personnel, 
mais sans l'ambition de quadriller le territoire entier, pour produire une image fidèle des lieux et de l'époque, plutôt qu'une émotion artistique décalée.

12 Au final, l'Atlas des Régions Naturelles est un beau projet artistique, qui nous surprend et stimule l'imagination, on essaye d'expliquer les choix de sujets des photographies, de les relier à la région concernée... La déconvenue de ne pas y découvrir une grande entreprise d'imagerie photographique des territoires est compensée par le plaisir de la découverte et l'appréciation de l'humour des artistes.

L'auteur souhaite remercier Anne-Christine Bronner pour ses remarques.

\section{BIBLIOGRAPHIE}

DEPARDON R. (2010). « La France de Raymond Depardon ». Paris : Le Seuil/BNF.

GROUPE M (1992). Traité du signe visuel, pour une rhétorique de l'image. Paris : Seuil.

TABUCHI É. (2019). « EDF - Électricité de France ». Paris : Poursuite.

LE TOQUIN CH., VOISIN L. (2018). « Trente ans de photographie de paysage en France : une histoire politique ». Métropolitiques, 1er février 2018. En ligne : https://www.metropolitiques.eu/Trenteans-de-photographie-de-paysage-en-France-une-histoire-politique.html

WARBURG A., RECHT R. (2010). L'atlas mnémosyne. Paris: Institut national d'histoire de l'art.

\section{AUTEUR}

\section{LAURENT JÉGOU}

Université de Toulouse-Jean Jaurès/UMR LISST-Cieu 